(Aus dem physiologischen İnstitut zu Königsberg i. Pr.)

\title{
Beiträge zur Physiologie des inneren Ohres.
}

\author{
Von \\ Dr. med. I. Streln. \\ (Enthält zugleich Beobachtungen von \\ L. Hermann, Fr. Matthias, M. Podack, P. Junius.)
}

Im Folgenden soll kurz über Untersuchungen berichtet werden, welche seit einer Reihe von Jahren auf Veranlassung and unter Leitung des Herrn Geheimrath $\mathrm{Hermann}$ ausgeflihrt worden sind, und an welchen ansser mir zeitweise anch andere Herren, besonders Herr Dr. Fr. M a t th i as, betheiligt waren.

\section{Die Schallreactionen an labyrinthlosen Tauben, sowie an taubstummen Menschen.}

Die höchst merkwürdige Beobachtung von J. R. Ewald ${ }^{\mathfrak{1}}$ ), sowie von Fano \& Masin $\mathrm{i}^{2}$ ), dass labyrinthlose Tauben noch auf Schall reagiren, würde, wenn sie auf Erregung des Acústicusstammes durch Schall beruhte, mit verbreiteten Vorstellungen uber allgemeine Nervenreize und specifische Sinnesreize in Conflict kommen, und ist daher vielfach mit grossem Misstrauen aufgenommen worden. Am weitesten ist wohl in dieser Richtung: Matte ${ }^{3}$ ) gegangen, welcher einfach die Thatsache bestreitet, indem er die Reflexe auf Schiisse als einzig sicheren Beweis der Schallwahrnehmung anerkennt, diese Reflexe aber bei der labyrinthlosen Taube vermisst. Das andere Extrem der Ansicht findet sich bei $\mathrm{Wundt^{4 }}$ ), welcher geradezu auch das normale Hören von directer

1) Berliner klin. Wochenschr. 1890. Nr. 32. Physiologisehe Untersuchungen über den Nervus octavus. Wiesbaden 1892. S. 24.

2) Centralbl. f. Physiol. Bd. 4. S. 25. 1891.

3) Dies Archiv Bd. 57. S. 437; s. auch Bernstein, ebendaselbst S. 475 .

4) Philosophische Studien Bd. 8. S. 641, Bd. 9. S. 496. 
Nervenfasererregung ableiten und den specifischen Endorganen nur eine klanganalytische Bedeutung zuerkennen will.

Die ersten hiesigen Beobachtungen in der vorliegenden Frage wurden von den Herren Hermann und Matthias im Sommer 1893 an einer von Herrn Prof. Ewald operirten und liebenswürdiger Weise nach Königsberg gesandten labyrinthlosen Taube gemacht, welche über 9 Monate lang im Institute lebte. (Eine gleichzeitig eingesandte einseitig labyrinthlose Taube lebt noch jetzt, Mai 1895.)

Seitdem hat zuerst Herr. Dr. Matthias, nachdem die von Ewald empfoblenen experimentellen Hülfsmittel beschafft waren, im Februar and März sowie im September 1894 einer Anzahl Tauben beide Labyrinthe exstirpirt; auch von diesen war eine im Februar 1894 operirte bis vor Kurzem am Leben. Endlich habe auch ich selbst im letzten Wintersemester mehrfach die Operation nit Erfolg ausgefiuhrt. Eine beiderseits von mir operirte Taube lebt ebenfalls noch jetzt (Mai 1895).

Es sei hier bemerkt, dass die von $\mathrm{Ewald}$ vorgeschriebenen complicirten Proceduren, mögen sie auch theilweise zuerst wie übertrieben ängstlich erscheinen, sich als unentbebrlich herausgestellt haben, und dass wir keinen Theil des Instrumentariums, weder den Taubenhalter noch die Westien'sche Lupe, die Instrumente zum Aufbrechen des Schädels, den Galvanocauter etc. entbehren möchten, wie auch die übrigen Vorschriften, bezüglich der Fütterung u. dgl., sich als sebr zweckmässig bewährt baben. Statt der Beleuchtung mit Lampe und Spiegel benutze ich neuerdings eine an der Westien'schen Lupe angebrachte kleine Glühlampe, welche mit einem kleinen Accumulator betrieben wird.

An der Ewald'schen labyrinthlosen Taube haben die oben genánnten Herren 4 Tage nach Ankunft des Thieres, d. h. am 10. Juli 1893, mebrfach zweifellose Reaction auf Rufen, z. B. Uh, beobachtet. In späteren Versuchen, besonders mit Schüssen (Zündbuitchen verschiedenen, zum Theil sebr grossen Kalibers) trat dagegen meist Versagen ein, jedoch war damals das Versuchsziel ein anderes, und es wurde daher auf diese negativen Ergebnisse, welche auch an den von Hern Dr. Matthias operirten Thieren verzeichnet wurden, kein besonderer Werth gelegt, sondern die genanere Prüfung dieses Punktes einer späteren Zeit vorbehalten. Wäre aber damals Anlass zu einer öffentlichen Aensserung 
gewesen, so würde sie überwiegend negativ ausgefallen sein, ähnlich der späteren von Matte.

Als ich selber endlich die im Institut vorbandenen, theils von Matthias, theils von mir operirten labyrinthlosen Tauben auf Schallreactionen untersuchte, gelangte ich bald zu durchaus sicheren und regelmässigen Ergebnissen, auch an denjenigen Thieren, an welchen friiher negative Resultate erhalten worden waren. Der Gesichtssin n war bei allen meinen Versuchen durch die Ewald'sche Kopfkappe ausgeschlossen, was für das Gelïngen sehr wesentlich ist. A uf Schüsse erhält man allerdings fast niemals eine deutliche Reaction, wohl aber auf Töne, besonders solehe von Lippen- oder Zangenpfeifen ${ }^{1}$ ).

Die Reaction besteht nicht in dem wohl meist erwarteten Zusammenschrecken, sondern hat einen ganz anderen, und zwar sehr regelmässigen Character. Sie tritt bei Tage ebensogut wie in der Stille der Nacht ein; ich fand es nicht nöthig, wie die Herren Hermann und Matthias auf Grund einer Angabe Ewald's häufig gethan hatten, die Nachtruhe zur Beobachtuing einer Reaction zu opfern.

Das Thier reckt auf jeden Schall der angegebenen Art in höchst characteristischer Weise Hals und Kopf ror und in die Höhe, unter leichtem Hin- und Herschuitteln. Die Bewegnng hat" durchaus nicht den Anschein eines erschrockenen Zusammenfahrens sondern eher einer Reaction auf eine unangenehme Empfindung. Auch sind grade plöt $\mathrm{l} l \mathrm{i}$ c h e, kurz vorübergehende, selbst sehr heftige, knallartige Schalle, wie Schïsse, Händeklatschen, Hammerschläge, wie schon bemerkt, o h n e Wirkung.

Der Schall braucht weder sehr heftig noch sehr nahe zu sein, um die erwähnte Reaction hervorzurufen; man kann sie mit einer Pfeife, Telephontrompete u. dgl. vom Nachbarzimmer aus bewirken, wenn die Thür nicht völlig geschlossen ist. Der Einwand, dass der zum Anblasen der Pfeife dienende Luftstrom das Thier direct treffe, wird schon hierdurch ausgeschlossen, noch besser freilich dureh besonders von mir angestellte Versuche. Die

1) Die Thatsache, dass auf Schüsse keine sichere Reaction auftritt, haben wir schon vor dem Erscheinen von Ewald's letzter Publication, welche denselben Umstand hervorhebt (dies Archiv Bd. 59. S. 271), constatirt. 
Reaction blieb nicht ans, wenn zwischen Taube und Pfeife ein grosser Schirm anfgestellt wurde, und liess sich andrerseits durch directes schallloses Blasen durch eine Kautschuk- oder Pappröhre nicht hervorrufen, selbst wenn der Luftstrom das Thier direct und aus nächster Nähe traf.

Unzweifelhaft also reagiren labyrintblose Tauben auf gewisse vibrirende tonerzengende B e w e g u g e n. Die zweite Frage ist, ob diese Reaction wirklich auf einer Erregung der Acusticusstümpfe beruht, wie $\mathrm{E}$ wald behauptet.

An sich wäre eine solche Erklärung für diejenigen Physiologen nichts Unerhörtes, welche überhaupt die Schallerregung auf eine „mechanische Tetanisirung: wollen, also auf einen allgemeinen Nervenreiz, für den sie allerdings den Nervenendigungen einen höheren, specifischen Grad von Erregbarkeit zuschreiben. Aber Ewald's Beweis für seine Erklärung hat eine sehr schwache Seite. Er behauptet nämlich, dass, wenn man die Acusticusstimpfe durch Einbringen von Arsenikpaste oder Krotonöl in die Knochenkanäle zerstört, die Schallreaction verhindert wird. Durch diese Operation kann doch wohl nicht der ganze centrale Rest der Acusticusfasern vernichtet werden; wenigstens ist nicht bekannt, dass caustische Zerstörung eines Nervenabschnittes auch den nicht direct betroffenen Rest des Nerven afficirt. Beruht aber die Zerstörung auf Ausbreitung des Aetzmittels selbst bis zu den centralen Enden des Nerven, dann würde eine so intensive Hirnläsion vorliegen, dass ein Functionsausfall irgend welcher Art nichts für Localisirung der Function beweisen würde. Warum aber die in der Hirnșubstanz liegenden Acusticusreste nicht eben so gut vom Schall gereizt werden sollen, wie die in den Knochenkanälen liegenden, ist nicht recht zu verstehen ${ }^{1}$ ). Ein noch ernsterer Einwand liegt in dem Umstande, dass die Schallreaction noch viele Monate nach der Labyrinthexstirpation beobachtet wird, ja sogar, wie es mir nach meinen

1) Ewald hat neuerdings angegeben (dies Archiv Bd. 59. S. 274), dass ihm der Aetzversuch nicht mehr gelingt, also vielleicht überhaupt nur einmal oder wenigstens in sehr wenigen Fällen gelungen ist. Dieser Umstand scheint mir um so mehr ein Hinderniss, ihm eine entscheidende Bedeutung in einer so fundamentalen Frage beizulegen. 
Versuchen scheint, mit der Zeit sicherer und energischer, während man doch annehmen muss, dass die centralen Acusticusstümpfe nach Abtrennung ihres Spinalganglions der D e g e n e ration anheimfallen, wie andere sensible Nerven ${ }^{1}$ ); als Spinalganglion des Acusticus wird aber für den Schneckenast das Ganglion spirale betrachtet; mag auch für den Vestibularast das trophische Centrum mehr central liegen, die Vestibularnerven also nach Labyrinthexstirpation möglicherweise von der Degeneration verschont bleiben : hier handelt es sich um die das Hören vermittelnden Nerveñfasern, d. b. nach $\mathrm{E}$ w ald um die Schneckennerven. Ueber ihre Degeneration kann kein Zweifel sein.

An einigen Gebirnen von Tauben, deren Labyrinth längere Zeit vor dem Tode exstirpirt war, babe ich den centralen Verlauf des Acusticus auf degenerirte Fasern untersucht, und glaube in der That solche gefunden zu haben; da aber eine umfassendere Feststellung wïnschenswerth erschien, so hat Herr Privatdocent Dr. Askanazy ein solches Gehirn zu untersuchen sich freundlichst bereit erklärt; über das Ergebniss wird nachträglich berichtet werden.

Ist nun wirklich eine Ableitung der Schallreaction von Empfindungen, welche durch andere Nerven als die Acustici vermittelt werden, ansgeschlossen? E.w ald giebt an, dass die labyrinthlosen Tauben noch reagiren, wenn man ihnen die Trommelfelle zerstört, die Federn abschneidet, und sie auf Watte stellt. Ich kann alle diese Angaben, soweit ich sie prüfen konnte, bestätigen, halte sie aber keineswegs für entscheidend.

Dass vor Allem Schallsehwingungen im Stande sind, vom Tastsinn empfunden zu werden, ist aus zahlreichen Erfahrungen bekannt. Je directer die Hànd mit dem schwingenden Körper verbunden ist, um so lebhafter ist die vibratorische Empfindung. Sehr bemerkenswerth sind z. B. folgende, theils von Herrn Prof. Hermann, theils von mir selbst gemachte Beobachtungen. Wird eine auf einem Resonanzkasten befestigte Stimmgabel angeschlagen oder angestrichen, so füblt man die Schwingungen sehr deutlich, wenn man die Finger an den Resonanzkasten hält. Je tiefer die Gabel,

1) Dieser Einwand, auf den Herr Prof. Her man n mich schon im Beginn meiner Arbeit im J. 1891 aufmerksam gemacht hat, ist seitdem in der im Sommer 1894 erschienenen Arbeit von Matte ausführlich entwickelt. 
um so kräftiger ist die Empfindung. Sitzt man anf einem Stuhl, so fühlt man seine eigene Stimme deutlich an der Lehne, sobald man sich anlehnt, besonders auch an der Seitenlebne eines Schreibtischsessels, sobald man denselben hinten mit dem Rücken berührt; es ist der Fremitus pectoralis, welcher sich dem Holz mittheilt. Aber auch durch die Luft fortgepflanzte Schwingungen setzen geeignete Körper in leicht fühlbare Mitschwingung. An der Membran eines König'schen Phonautographen fühle ich, sowie Hr. Prof. H e r m a n n, in geschlossenen Räumen Männerstimmen, besonders Vocal $I$, bis über 8 , eine Zungenpfeife $c^{2}$ bis über 31 Meter! Das Länten grosser Kirchenglocken, welche $2 \mathrm{~m}$ über dem Erdboden aufgestellt sind, fühle ich an einem ibber einen Rahmen gespannten Papierblatt im Freien bis $25 \mathrm{~m}$, und unmittelbar an meinem Körper noch viel weiter.

Sehr bemerkenswerth ist, dass beim Fühlen von Stimmgabeln die directeste Zuleitung der Schwingungen nicht immer die günstigste ist. Beispielsweise finde ich, dass eine angeschlagene Stimmgabel viel deutlicher gefuihlt wird, wenn man ihren Stiel auf die mit dicker Kleidung bedeckte Brust setzt, als wenn man sie auf die nackte Brust oder, auf den Kopf hält. Ebenso füblt man die Vibrationen einer angeschlagenen $c^{1}$-Stimmgabel $\left(U t_{3}\right)$ nicht 'so gut, wenn man ihren Stiel auf die nackte Fingerkuppe hält, als wenn sich zwischen Finger und Gabel ein zusammengelegtes Handtuch oder eine mehrfache Wattelage befindet. Im letzteren Falle fii h l t man das Schwirren noch sebr deutlich, wenn die Schwingungen so weit verklungen sind, dass man sie bei diesem Abstande vom Ohre nicht mehr deutlich $\mathrm{h} \ddot{\mathrm{o}} \mathrm{r}$ t.

Wie wenig dicke Wattelagen die Mittheilung von Schwingungen hindern, habe ich bei Microphonversuchen constatirt, welche ich angestellt habe als Vorversuche zur acustischen Isolation des Taubenkörpers. Verwendet wurde ein Microphon neuerer Construction, wie es zum Telephonverkehr benutzt wird, bestehend aus 4 von Filzringen umfassten Kohlengrusslagern zwischen zwei Kobleplatten. Der von einem $\mathrm{D}$ a n i ell gelieferte, durch das Mierophon gehende Strom wurde einer primären Spirale zugeleitet, und die secundäre Spirale war mit einem in einem entfernten Zimmer befindlichen Siemens-Telephon verbinden; letzteres war durch eine Nebenschliessung ( $\mathrm{d}$ u - B o i s'scher Schliissel) absperrbar, um leise Töne sicherer zu constatiren. Pfeifentöne hört man 
in diesem Falle am lautesten, wenn das Microphon anf der nackten Tischplatte liegt, weniger laut, wenn es frei schwebt, oder auf eine dicke auf den Tisch gelegte Watteschicht gebracht wird, aber man hört auch dann noch zweifellos, wenn auch sehr schwach, wenn das Microphon in dicke Watte ganz eingepackt, und so in einen mit Kautsehnk bedeckten Cylinder gesteckt wird. Das, Microphon dürfte ein Apparat genannt werden können, welcher bezüglich der Wahrnehmung von Vibrationen zwischen der fühlenden Fingerkuppe und dem Ohre steht.

So gering die Aussicht war, durch mechanische Isolation die Sehwingungen von irgend einem Körpertheil der Taube völlig àbzuhalten, musste sich doch durch Erschwerung der Zuleitung, entweder zum Kopf oder zum Körper der labyrintblosen Taube, eine Andentung ergeben, welcher der beiden Theile die Reaction hauptsächlich vermittelt. Da Einpackungen des Kopfes sich schon deswegen verbieten, weil an ihm die Reaction fast allein beobachtet werden kann, konnte es sich nur um Erschwerung der Zuleitung zum Körper handeln. Ich benutzte dazu Einpackung in Watte und Versenken in Oel. In den ersteren Versuchen befand sich das Thier in einem an der Decke aufgehängten und durch einige am Fussboden befestigte Fäden an Drehungen verhinderten Drahtkäfig, dessen Boden mit Watte ausgelegt war, ïber welche ein Stiick Raumwollenstoff gespannt ist. Für die Reactionen zeigte es sich nun ganz zweifellos von entscheidendem Einfluss, ob der Körper mit Ausschluss des Kopfes mit Watte eingepackt war oder nicht. Im letzteren Falle reagirte die Taube regelmässig auf grosse Entfernungen, im ersteren entweder ïberhaupt nicht, oder nur aus allernächster Näbe. Da die Schallwellen den Kopf in beiden Fällen gleich gnt erreichen, so ist es zum mindesten höchst unwahrscheinlich, dass die Reaction anf Erregung der Acusticnsstïmpfe berulat. Dasselbe Resultat hatten die Oelversuche. Ein sehr grosses cylindrisches Glasgefäss von $80 \mathrm{~cm}$ innerer Höbe und $30 \mathrm{~cm}$ innerem Durchmesser wurde zum grösseren Theil mit zimmerwarmem Wasser, und dariber $15 \mathrm{~cm}$ hoch mit ebenfalls zimmerwarmem Maschinenöl gefüllt. Die Taube wurde (mit Kopfkappe) mit zusammengebundenen Flügeln in das. Oel eingelegt, in welchem sie sich schwimmend hält, und zwar in normaler Haltung, wenn der Schwanz dureh ein Gewicht von $10 \mathrm{gr}$ beschwert ist. Der Kopf wurde mittels eines Fadens an einem über 
den Rand des Gefässes gelegten Holzstabe befestigt. Im Freien ähnlich gebunden und befestigt reagirt das Thier wie gewöhnlich auf grosse Entfernungen, im Oel dagegen nur, wenn der Schall in unmittelbarster Nähe erzeugt wird. Dieser Versuch führt zu demselben Schlusse, wie der Einpackungsversuch. Wiederholte Oelversuche schädigen, beiläufig bemerkt, die Tauben dauernd.

Besonders gut reagiren die Tauben, wenn man sie auf ein an Drähten aufgebängtes dünnes Brett von Cigarrenkistenholz stellt. Sie reagiren hier am stärksten, wenn man mit einem schwingenden Körper, z. B. einer angeschlagenen grösseren Stimmgabel, das Brett von unten her berihrt, sei es mit dem Stiel, sei es, was besonders wirksam ist, mit einer Zinke. Die Reaction besteht immer in dem schon beschriebenen characteristischen Erheben und Schütteln des Kopfes. Legt man dicke Watteschichten unter die Füsse, so hat dies zwar einen schwächenden Einfluss auf die Reaction, verhindert sie aber nicht.

Diese Erfahrungen beguinstigen die Vermuthung, dass die Schallreaction labyrinthloser Tauben auf einer tactilen Wahrnehmung der vibrirenden Bewegung beruht, vielleicht auf einer dem Thiere nnangenehmen oder es erschreckenden Kitzelempfindung. Beachtung verdient übrigens, dass die $\mathrm{L} u \mathrm{fts}$ ä cke der Taube möglicherweise die Einwirkung von Vibrationen auf das Gefuihl sehr begünstigen.

Es lag nahe, den Gedanken der tactilen Schallwahrnehmung auch an taubstummen M ens chen zu prïfen, welche über ihre Wahrnehmungen Aufschluss geben können. Ich liess daher die beiden taubsten Schüler einer der hiesigen Taubstummenanstalten ${ }^{1}$ ) in das Institut kommen; sie wurden von einem Lehrer begleitet, welcher sich ausgezeichnet mit ibnen verständigen konnte. Der eine Knabe A. B. ist 13 Jahre alt, und seit dem 7. Jahre gänzlich taub, der zweite G. W. 12 Jahre alt, gänzlich taub seit dem 3. Lebensjahre.

Beide Schïler, mit wollenen Strümpfen und Stiefeln, stehen etwa 2 Meter vom Untersuchenden mit abgewandtem Gesicht auf dem Fussboden, und haben bei mehrmaligem Anblasen einer Zungen-, resp. Lippenpfeife, die Zahl der wahrgenommenen Töne anzugeben. Die Zahl wird für die Zungenpfeife von beiden richtig angegeben; beide behaupten in vielen Fällen, aber nicht durchweg, die Empfin-

1) Dieselbe hat etwa 90 Schüler. 
dung in den Füssen zu haben. Die Lippenpfeife wird von B. nicht, von W. richtig wahrgenommen.

Weitere Versuche wurden mit begünstigter Zuleitung vorgenommien, indem die Knaben mit blossen Füssen auf einem Tisch standen. Bei beiden Verfahren werden von W. stets richtige Angaben gemacht, von B. sehr unsichere und oft falsche. W. empfand den Sehall auch bei erschwerter tactiler Zuleitung, indem er in einen an der Decke aufgehängten gepolsterten Riemen gesetzt wurde, den er mit den Händen nicht anfassen durfte.

W. nimmt iberhaupt besser wahr als B., der einen etwas stumpfsinnigen Eindruck macht. Werden. Zündhütchen durch Hammerschläge auf einem Ambos verknallt, wobei in der Regel einige Schläge versagen, so geben beide die Zahl der Hammerschläge richtig an, W: empfindet ausserdem den letzten, die Explosion erzeugenden, stärker als die Fehlschläge. Auf die Frage, mit welchem Körpertheil die Schläge wahrgenommen werden, werden zuweilen die Füsse, häufiger aber die Kopfgegend hinter den Ohren, oder auch nur ein Ohr bezeichnet.

Der begleitende Taubstummenlehrer giebt an, dass die Schüler weit feinfühliger sind als er. Wenn beim Unterricht ein Wagen durch die vom Gebäude etwas entfernte Strasse fährt, den er weder hört noch fühlt, so sehen sich die Schüler nach demselben $\left.\mathbf{u m}^{1}\right)$. Aehnlich wie bei Blinden scheint auch bei Tauben der Tastsinn sich zu verfeinern, wenn auch in anderer Richtung. Dass anch die labyrinthlosen Tauben Schallreactionen lange Zeit nach der Operation sicherer zeigen, als in den ersten Wochen, habe ich schon erwähnt.

Beide Schüler waren schon $1 \frac{1}{2}$ Jahre vorher bei einer Gesammtuntersuchung (s. unten) auf Drehschwindel und galvanischen Schwindel geprïft worden, und diese Prüfung wurde jetzt wiederholt. B. zeigte damals und jetzt Drehschwindel und rotatorischen Nystagmus; der galvanische Schwindel und Nystagmus war früher vermisst worden, war aber jetzt vorhanden. W. hatte beide Male auf dem Drehstuhl weder Schwindel noch Nystagmus; der galvanische Schwindel hatte früher gefehlt, während Nystagmus aufgetreten war; jetzt war beides vorhanden. Diese Verschiedenheit

1) Ein anderer hierüber befragter Taubstummenlehrer hat hiervon nichts bemerkt, was vorsichtshalber ausdrücklich erwähnt wird. 
des Ergebnisses in $1 \frac{1}{2}$, jährigem Intervall scheint mir von grossem Interesse.

Auch aus der zweiten hiesigen Taubstummenanstalt liess ich die beiden taubsten Schiiler in das Institut kommen, einen 15jährigen Knaben C. W., seit dem l. Lebensjahre taub, und ein 13jähriges Mädchen A. W., seit dem 8. Jahre taub. Der Knabe empfindet, auch im Riemen sitzend, Pf́ifentöne regelmässig; als Ort der Empfindung giebt er die Gegend hinter dem Ohre an. Scbüsse nimmt er auch entfernt wahr, wie, ist nicht festzustellen. Das Mädchen empfindet nur tiefe Pfeifentöne. Ein Schuss in der Nähe macht ihr Schmerz im Ohr, aber keine eigentliche Schallempfindung; in der Entfernung (Nebenzimmer) nimmt sie Nichts davon wahr.

Die Untersuchung auf Drehschwindel giebt bei beiden positive Resultate. Galvanischen Schwindel zeigt nur der Knabe, Nystagmus beide. Das Mädchen war schon vor $1^{1 / 2}$ Jahren untersucht worden, und hatte damals anch galvanischen Schwindel gezeigt.

Im Ganzen schliesse ich aus meinen Versuchen, dass es kcineswegs nachgewiesen ist, dass die Schallempfindungen bei Labyrintbmangel von den Acusticusstümpfen herrühren. Vielmehr erscheint es sehr wohl möglicb, dass es sich nur um tactile Empfindungen handelt, für welche vielleicht eine grössere Erregbarkeit sich anerzogen hat.

Neuerdings hat Ewald ${ }^{1}$ ), veranlasst durch die Veröffentlichungen von Matte und Bernstein, sich noch einmal über die vorliegende Frage geäussert. Neben manchen Bemerkungen, welche mit unseren Eq̣gebnissen völlig übereinstimmen, enthält diese Abhandlung eine Anzahl abweichender Angaben. So behauptet Ewald, dass die Tauben anf Anstreichen der Unterlage mit dem Violinbogen nicht reagiren; dieser Widerspruch löst sich vielleicht dadurch, dass $\mathrm{Ew}$ a ld nur solide Tischplatten, ich aber auch dünne Grundbretter angestrichen habe. Eine weit stärkere Differenz liegt in dem Umstande, dass die Reactionen bei Ewald nur Nachts, bei mir aber ausgezeichnet und regelmässig auch am Tage auftraten. Dies könnte dem Gedanken Raum geben, dass es sich bei den beiderseitigen Beobachtungen um verschiedene Arten der Reaction handelt, und Ewald die von mir beschriebene characte-

1) Dies Archiv Bd. 59, S. 258. 
ristische Vibrations - Reaction nicht beobachtet hat, wir aber nicht dasjenige, was ihm als Schallreaction erscheint. Auch davon, dass die Reaction noch viele Monate nach der Exstirpation des Iabyrinthes, ja weit sicherer als anfangs auftritt, findet sich bei $E$ wald Nichts. Er hat schon am Abend nach der Operation die Reaction festgestellt. Dass er sie freilich auch in späteren Zeiten nicht vermisste, folgt schon aus dem Umstande, dass er solche Tauben Monate nach der Operation zur Constatirung der Schallreaction versandte. Die unserm Institut am 6. Juli 1894 zugegangene Taube war z. B. rechts am 13. April, links am 29. Mai operirt. Um so mehr darf ich mein Befremden aussprechen, dass $\mathrm{E}$ w al d gegen den schon von Matte erhobenen Einwand der Degeneration nur das anfiuhrt, dass die Schallreaction schon am Abend nach der Exstirpation vorhanden ist.

Bei einem so zuverlässigen Beobachter wie Ewald muss ich immerbin die Möglichkeit anerkennen, dass wir, wie schon angedentet, vielleicht ganz verschiedene Erscheinungen als Schallreaction betrachten. Die von mir beobachtete ist aber unzweifelhaft zu einer Zeit vorhanden, wo die Acusticusreste degenerirt sein müssen, und aus den erwähnten Zeitangaben scheint hervorzugehen, dass dies auch für $\mathrm{E} w$ ald 's Schallreaction angenommen werden muss.

\section{Ueber den angeblichen Zusammenhang des galvanischen Schwindels mit dem Labyrinth.}

Während im hiesigen Institut $\mathrm{E}$ wald's A n gaben ii ber das Verhalten, labyrinthloser. Tauben in fast allen Beriebugen, theils an den von ihmeingesandten, theilsanden hieroperirten Thieren bestätigt worden sind, besteht eine wesentliche Differenz hinsichtlich der Reaction auf transversale Kopfdurehströmung. Ich habe die Frage ihres Zusammenhanges mit den Bogengängen in zahlreichen Versuchen an Fröschen, Tauben und Menschen, welche bis in das Jahr 1892 zurückreichen, bearbeitet, und will im Folgenden die Ergebnisse kurz mittheilen.

Frösche, auf einer Platte sitzend, zeigen auf mässige Ströme (2 kleine Chromsäure-Elemente), welche man mittels kleiner in die Trommelfelle eingehakter Drahtösen durch den Kopf leitet, regel- 
mässig Torsion des Kopfes und des vorderen Theiles der Wirbelsäule nach der Anode. Die Labyrinthe exstirpirte ich zum Theil nach Schrader's Vorgang von der Mundhöhle aus, häufiger aber nach folgendem etwas bequemeren Verfahren, von aussen her. Ich spaltete die Hant über dem Os petrosum von einem. Trommelfell bis zum anderen, dann machte ich einen Medianschnitt, bog die entstandenen vier Hautlappen zurïck und beschwerte sie dureh herabhängende kleine Klemmen, da sie bei Bewegungen des Frosches, der auf dem Bauche liegend angebunden ist, leicht zurịckklappen. In diesem so entstandenen viereckigen Felde sieht man vor sich zuerst die Fascie; diese wird vorsichtig entfernt, obne die darunter liegende und durchschimmernde Arteria occipitalis zu verletzen. Dann wird die Arterie durchschnitten, nachdem man sie durch Compression neben der Wirbelsäule blatleer gemacht hat. Doch da es unbequem ist, während der ganzen Operation mit der einen Hand zu comprimiren, und die Blutung bisweilen am anderen Ende der Arterie noeh störend auftritt, ist es besser, die Blutung mit dem Galvanocauter zu stillen. Dann löst man den Musculus temporalis von seiner oberen Insertion ab, klappt ihn zurïck und hat so das Felsenbein vor sich liegen. Nachdem man sich am knöchernen Präparat genügend orientirt hat, bohrt man mit der Messerspitze eine Oeffnung in das Felsenbein und erweitert diese, bis man bequem mit einer kleinen Pincette eingehen kann. Es ist zweckmässig, zuerst mit. einer Nadel das Otolithen-Säckchen zu öffnen und mit einer fein gezogenen Glasröhre die OtolithenKryställchen herauszusaugen und dann erst mit der Pincette das Labyrinth zu fassen. Man ïberzeugt sich davon, ob das ganze Labyrinth entfernt ist, dadurch, dass man in einem Uhrschälehen in Wasser genau untersucht, ob es vollständig ist. Nur in diesem Falle wurde die Exstirpation als gelungen betrachtet. Nachdem auf der anderen Seite ebenfalls das Labyrinth exstirpirt war, wurden die Musculi temporales wieder an den Knochen herangelegt, die Fascie darüber, und dann die Hautlappen durch mehrere Knopfnähte wieder vereinigt.

Ein beiderseits operirter Frosch reagirt sicher auf den galvanischen Strom, vielleicht noch stärker als ein gesunder. Ein anf einer Seite frisch operirter Frosch reagirt ebenso constant auf den Strom wie ein normaler, doch scheint die Bewegung lebhafter zu sein, wenn die Cathode an der operirten Seite liegt. 
An der von Ewald unserm Institut freundlichst zugesandten' beiderseits labyrinthlosen $\mathrm{T}$ a a b e hatte schon Herr Prof. Herman $n$ in Gemeinschaft mit Herrn Dr. Matthias Versuche über galvanische Reaction angestellt. $Z u$ denselben diente ein von. Herrn Prof. Hermann angefertigter sehr leichter und einfacher Electrodenhalter von folgender Construction. Ein federnder Eisendraht ist halbkreisförmig so gebogen, dass er un den Kopf der Taube berumgeht; das eine Ende ist reclutwinklig in frontaler Richtung nach innen umgebogen, so dass es in das eine Ohr gesteckt werden kann, und endet knopfformig; das aadere Ende ist um ein Holzpföckehen herumgewunden, welches ebenfalls frontal steht, und durch welches ein kurzes ebenfalls knopfförmiges Eisendrähtchen gesteckt ist. Von den beiden dünnen kupfernen Zuleitungsdrähten ist der eine an das letztgenannte Eisendrältchen, der andere an den. Bügeldraht selbst, an dessen Umbiegungsstelle gelöthet. In beide Gehörgänge werden winzige mit Kochsalzlösung getränkte Schwämmchen gesteckt, in welche die beiden knopfförmigen Draht. enden federnd sich eindrïcken. Der Bügel liegt stets tadellos fest.

Die Tauben stehen meist, mit der Ewald'schen Kopfkappe versehen, frei auf dem Tisch; zuweilen wurden sie auch in ein Handtuch eingewickelt, das nur den Kopf frei liess, beobachtet. Sowohl an der Ew ald'sehen Taube wie an den von Herrn Dr. Mattbias und von mir operirten Thieren wurde regelmässig Neigen des Kopfes nach der Anodenseite beobachtet, und es bedurfte hierzu durchaus keiner stärkeren Ströme als bei gesunden. An der von Herrn Professor. E w a ld hierher gesandten einseitig labyrinthlosen Taube trat die Reaction etwas stärker ein, wenn die Cathode auf der unverletzten Seite, als wenn sie auf der verletzten lag, feblte aber auch in diesem Falle nicht. Auch bei der Oeffung, besonders nach längerer Sehliessung, sieht man oft die Gegenreaction nach der Catbode ${ }^{1}$ ). Di e Strom richtung läs st sich aus den Reactionen stets mit Sicherheit entnehmen.

1) Hinsichtlich der Gegenwirkung bei der Oeffnung macht $\mathbf{E w}$ a $1 \mathbf{d}$ (N. octavus S. 238) die Bemerkung, die Polarisation sei dabei betheiligt, weil die Gegenwirkung bei Anwendung unpolarisirbarer Electroden geringer ausfällt. Hier liegt anscheinend ein Irrthum zu Grunde. Polarisation an den Electroden könnte höchstens dann einen Einfluss haben, wenn die Oeffnung jenseits einer Nebenschliessung stattfände, so dass der Polari- 
Dies einfache, oft constatirte Resultat steht nun anscheinend zu Ewald's Angaben in directem Gegensatz. Derselbe beobachtete érstens an labyrinthlosen Tauben auch mit den stärksten Strömen niemals die Reaction (N. octavus S. 232), sondern nur Zuckungen des Kopfes und anderer Muskeln, bei denen er vou eizer orientirten Beweging Nichts angiebt, zweitens an einseitig. labyrintblosen Tauben (S. 233) nur dann die gewöbnliche Reaction, wenn die Cathode am gesunden Ohre liegt; im anderen Falle "kommt keine starke Kopfneigung mebr zu Stande". Aus dieser Ausdrucksweise ist zu entnehmen, dass doch anch bier Kopfneigung beobachtet wurde, so dass eine Differenz gegen unsern Befund hier kaum vorhanden scheint. Vermuthlich erklärt Ewald diese Reaction als "Anodenhemmungswirkung" anf das noch vorhandene Labyrintb.

Die "Nebenreaction“, von welcher Ewald spricht (z. B. S. 235), kann nicht zur Erklärung unsrer Befunde herangezogen werden, da sie nach $\mathrm{Ew}$ ald keine orientirte zu sein scheint. Wäre sie das, d. h. macht das Thier auch dureb Reizung anderer Theile als Labyrinth und Acusticus Bewegungen nach der Anodenseite, so wäre jedenfalls der Behauptung, dass die eigentliche Reaction auf Labyrinthreizung berubt, der Boden fast entzogen.

Die zahlreichen feinen und sinnreich modificirten Versuche Ewald's mit Anbringung nur Einer Electrode am Ohr, sowie mit Anbringung beider Electroden an einem Labyrinth, oder Theilen desselben, enthalten bei näherer Prüfung keinen entscheidenden Beweis fur die behauptete Bezichung der Reaction zum Labyrintb. Denn nirgends ist die Mitdurchströmung des Gehims völlig ausgeschlossen. Etwas anderes wäre es, wenn es $\mathrm{E}$ wald gelungen wäre, durcb localisirte Reizung einzelner Bogengänge oder Ampulleu Bewegungen in den Ebenen derselben hervorzurufen, was aber nicht' der Fall ist; im Gegentheil tritt er den beziiglichen Bebauptungen Breuer's entschieden entgegen. Er giebt selbst an, dass in diesen Versuchen stets das ganze Labyrinth gereizt warde, und es ist nur hinzuzusetzen, dass stets anch das Gehirn Strom-

sationsstrom sich durch den. Kopf abgleichen kann; von einer Nebenschliessung spricht aber Ewald nicht. Handelt es sich aber um innere Polarisation in den Organen, so muss es gleichgültig sein, ob die Electroden polarisirbar sind oder nicht. 
antheile' erhalten musste. So beantwortet sich auch sehr einfach Ew al d's Frage (S. 249), warum auch die stärksten Ströme bei wirksamster A nordnung, für eine Ampulle „einen so relativ schwachen Erfolg zeigen". Er selber macht die höchst unwahrscheinliche Annahme, "dass der durch die electrische Frregung erzeugte Zustand der Nervenfasern im Oetavusstamm von einer Faser auf die andere iibergeht", und findet es wahrscheinlich (?), dass der Angriffspunkt für den Strom gar nicht das eigentliche. Endorgan, sondern die letzten Verzweigungen des Nerven selbst seien.

In Wirklichkeit scheint mir die Erklärung der Reaction durch Wirkung auf das Labyrinth, selbst wenn dieselbe nicht durch die Erfolge an labyrintblosen Thieren widerlegt wäre, ungemein schwierig, ja unmöglich. Man denke sich den Kopf der Taube, oder auch des Menschen von queren Stromfäden durchflossen, oder besser, man nehme gute Präparate zu Hülfe. Man wird dann erkennen, dass die durch die Ampullen gehenden Antheile die einzelnen Ampullen in den versehiedensten. Richtungen, wenn man die Crista acustica oder die Nerveneintrittsstelle zum Orientirungscentrum nimmt, durchsetzen. Jede Ampulle hat offenbar ibre Anoden- und Cathodenstellen, deren Dichte bei einem so tief in den Schädel geriickten oder wenigstens - was auf dasselbe herauskommt - von den Electrodenpunkten so weit entfernten Organ im Weșentlichen gleich sein muss. Unter diesen Umständen verstehe ich nicht, wie man sich mit Erklärungen wie Anodenwirkung an dem einen und Cathodenwirkung an dem anderen Labyrinth begniigen kann. Jede Ampulle muss Anodenund Cathodenwirkung zugleich empfangen. Höchstens also könnte man annehmen, dass der Erfolg eine Art resultirender Wirkung aller Ampullendurchströmungen wäre; auch dann aber würde ich Begriffe wie Cathodenreizung, Anodenhemmung, welche an das electrotonische Gesetz erinnern, nicht hineinwerfen, da sie von der äusseren Electrode hergenommen sind, und Niemand wissen kann, ob in der Resultirenden Anoden- oder Cathodenwirkung der Ampullen überwiegt. Wie ungemein unwahrs che in li ch ist es aber vollends, dass die resultirende Wirkung aller Ampullendurchströmungen bei so verschiedenen Geschöpfen wie Frosch, Taube, Kaninchen, Mensch stets dieselbe sein soll. Die Lage der physiologischen Anoden und Cathoden an jeder Ampulle muss doch nicht blos von der bei den einzelnen Thierarten ganz verschiedenen 
Orientirung dieser Organe zur Verbindungslinie beider Electroden und zum System der Strömungslinien abhängen, sondern dies letztere auch von den leitungsverhältnissen im Schädel. Können diese aber verschiedener sein, als bei Taube und Mensch? Bei jener liegen die Canäle in einem lufterfüllten Raum, von Bälkehen getragen, bei diesem in der compactesten Knochenmasse!

Ein besserer Ausweg würde es sein, wenn man die Erfolge von der Durchströmung des Nerven selwer ahleiten wollte. Man kann nämlich mit ziemlicher Wahrscheinlichkeit sagen, dass der Acusticusstamm auf der Seite der Anode wesentlich anfsteigende, auf der Seite der Cathode wesentlich absteigende Componenten erhalten wird, was sich freilich auf die centralen und peripheren End i gu n ge n sicher nicht ausdehnen lässt. Der Kopf würde sich also durch absteigende Durchströmung eines Acusticus von der betreffenden Seite wegwenden, und durch aufsteigende Durchströmung derselben zuwenden, und letztere Wirkung die weniger starke sein. Diese Ableitung scheitert jedoch gerade bei Ewald an der Behauptung, dass Exstirpation des Labyrinthes die betreffende Reaction aufbebt; bei uns aber, die wir das nicht zugeben, daran, dass sie anch noch zu einer Zeit vorhanden ist, wo die Acusticusstämme längst degenerirt sein müssen.

Auf der anderen Seite bat es keine Schwierigkeiten, die Erscheinung auf Durchströmung des Gehirns selbst zu beziehen. Einmal wissen wir, dass Zwangsstellungen und $Z$ wangsbewegungen, ohne Zweifel verbunden mit Schwindelempfindungen, durch Verletzung der verschiedensten Hirntheile vom Kopfmark ab, Brïcke, Kleinhirn, Grosshirnstiele, hervorgebracht werden , können, sobald nur die Verletzung unsymmetrisch ist. Quere Durchströmung des Gehirns ist aber wie eine höclsst unsymmetrisehe zarte Verletzung zu betrachten; dass eine solche Schwindel, Zwangsstellungen und Zwangsbewegungen macht, ist sehr wahrscheinlich, obwohl es zur Zeit unmöglich ist, eine speciellere Erklärung oder eine Localisation der Einwirkung zu versuchen. Dass B reuer1) und E wald ${ }^{2}$ ) leichter Reaction erhalten, wenn sie die betreffende Electrode auf die Bogengänge bringen, als wenn sie sie dicht daneben durch den Knochen bindurch in den Seitentheil des Klein-

1) Dies Archiv Bd. 44. S. 143.

2) N. octavus S. 228. 
hirns einstechen, beweist nichts. Denn erstens ist es willkürlich; grade das Kleinbirn aus den auf unsymmetrischen Angriff Schwindelreaction gebenden Hirntheilen herauszugreifen, zweitens geht aus Breuer's Darstellung deutlich hervor, dass er auch von der in's Kleinhirn eingestochenen Electrode Reaction erhalten bat, wenn auch erst bei etwas stärkeren Strömen. Da man annehmen muss, dass die Electrodennadel bis auf die Spitze isolirt war, denn sonst wäre das Resultat geradeza unverständlich, so kann man den Versuch ebensogut als Beweis benutzen, dass directe Stromzuleitung zum Kleinhirn die Reaction hervorrufen kann. Aber muss denn überhaupt die electrische Wirkung dieselbe sein, wenn die Electrode punktförmig direct auf einen Hirntheil aufgesetzt wird, wie wenn die Stromfäden von einem entfernteren Punkte her in gleichmässigerer Vertheilung in einen grösseren Theil der Oberfläche eintreten?

Dass orientirte Durchströmung von Centralorganen orientirte Einstellung von Körpertheilen machen kann, offenbar unter Einfluss von Empfindungen, dafür haben wir ein schönes Beispiel in der von Hermann entdeckten Einstellung von Froschlarven und Fischen in durchströmtem Wasser ${ }^{1}$ ). Dass das Kopfende hier gegen die Anode gewendet wird, hat sogar eine gewisse Analogie mit der galvanischen Zwangsdrehung böherer Thiere, welche freilich nur oberfächlich ist und nur mit grosser Vorsicht verwendet werden darf.

Ich balte es also durchaus für möglich, dass der galvanische Schwindel von Stromwirkung auf das Gehirn selbst herrihht. Die Gleichgewichtsempfindung wird, von welchen Organen sie auch herrïhren möge, symmetrische Erregung voraussetzen; die geringste Asymmetrie der peripheren Erregung nicht blos, sondern auch der centralen Erregbarkeiten wird Störungen der Gleichgewichtsempfindung mit ihren Folgen bis zur Zwangsbewegung hervorbringen. So werden selbst schwache das Gehirn durchsetzende Stromschleifen, wenn sie nicht etwa rein parallel der Symmetrieebene sind, den Keim zu solchen Erscheinungen in sich haben. Solche Stromschleifen wird aber das Gehirn bei jeder Stromapplication an den Kopf erhalten müssen, mit Ausnahme des Falles, dass beide Electroden median liegen. In der. That entsteht am

1) Dies Archiv Bd. 37. S. 457 , Bd. 39. S. 414, Bd. 57. S. 391. 
Menschen Schwindel nicht allein wenn beide Electroden oder nur eine in der Ohrgegend, sondern auch wenn z. B. beide, jedoch in unsymmetrischer Lage, im Nacken angebracht werden, wenn nur der Strom stark genug ist. Wie weit sich die Stromschleifen erstrecken, erkennt man an dem fast stets sich einmischenden electrischen Geschmack und den meist vorhandenen optischen Erscheinungen. Selbst wenn, wie in gewissen Ewald'schen Versuchen, beide Electroden an demselben Labyrinth liegen, werden die hier grade auffallend schwachen Erscheinungen (s. oben) viel wahrscheinlicher von Hirnschleifen als von directer Wirkung auf das Labyrinth berrühren.

Man könnte einwenden, da doch einmal das Labyrinth Bewegungsempfindungen und deren Reactionen auslöst, sei es unzweifelhaft, dass anch electrische Beeinflussung desselben solche Folgen haben müsse, da die Organe auch bei diesem widernatürlichen Reiz mit ihrer specifischen Energie reagiren müssen. In der That mag ja ein soleher Antheil vorhanden sein, aber die Hauptsache kann er unmöglich ausmachen, da die Reaction, wie ich auf das Bestimmteste behaupte, auch bei labyrintblosen Thieren auftritt, und ferner habe ich oben auf die grosse Schwierigkeit hingewiesen, die Constanz des resultirenden Erfolges bei den verschiedenen VerhäItnissen der einzelnen Wirbelthierklassen zu verstehen, wenn es sich wirklich um Labyrinthwirkungen handelt. Endlich vermisst man bei Ewald einen wichtigen Versuch: sehr richtig giebt er an, dass beim Versuch auf eine einzelne Ampulle zu wirken, stets das ganze Labyrinth gereizt wird.' Da nun auf diesem Wege eine isolirte Wirknng nicht zu erzielen ist, bätte er doch versuchen miissen, Modificationen dadurch hervorzubringen, dass er die Reizung nach Zerstörung einer oder zweier Ampullen wiederholte, ein freilich schwieriger, aber bei seiner feinen Operationstechnik nicht von vornherein unausführbar erscheinender Versuch.

Ich komme nun endlich zu den galvanischen Versuchen an taubstummen Menschen, wie sie zuerst von James'), dann von Pollak ${ }^{2}$, und in sehr grosser Zahl, zum Theil schon vor Pollak,

1) Amer. Journ. of otology Bd. 4. 1882.

2) Dies Archiv Bd. 54. S. 188. 
und wiederholt, von mir selber angestellt sind. Ueber meine eigenen Versuche habe ich Folgendes zu berichten.

Es wurden mir die Schiiler der beiden hiesigen TaubstummenInstitute, des „Provinzial-Taubstummen-Instituts" und der „Königsberger Vereins-Taubstummenanstalt", von den Herren Directoren in der liebenswürdigsten Weise zur Verfügung gestellt, wofür ich den Herren anch an dieser Stelle meinen verbindlichsten Dank sage. Bei allen den zahlreichen und zeitraubenden Versuchen wurde ich in der freundlichsten Weise von den Herren Taubstummenlehrern nnterstïtzt, ohne deren Hülfe es mir häufig ganz unmöglich gewesen wäre, zu einem Resultat zu gelangen.

Die erste Untersuchung habe ich mit Hiilfe des Herrn Dr. P o d a ck im Sommer 1892 in den Taubstummen-Instituten selbst gemacht. Es wurde sehr darauf geachtet, dass die schon Untersuchten mit den noch zu Untersuchenden nicht in Verkehr treten und sie auf die zu erwartenden Erscheinungen vorbereiten konnten. Eine ganz gewaltige, häufig nicht zu ủberwindende Schwierigkeit bei diesen Untersuchungen ist der Umstand, dass den meisten Taubstummen der Begriff "Schwindel" absolut unbekannt ist. Wenn man sichtlich. von Schwindelgefithl ergriffene Kinder nach ihren Empfindungen befragt, so gibt das eine wohl an, dass sich die Gegenstände mit ihm bewegt bätten, oder ein anderes vergleicht seinen Zustand mit dem eines Betrunkenen, ein drittes aber steht stumpfsinnig da, gibt auf alle Fragen verneinende Antworten und macht den Eindruck, als ob ibm sein Zustand gar nicht zum Bewusstsein gekommen sei; ein viertes will mit deutlichem Ausdruck der Furcht vor etwas ihm Unerklärlichen davonlaufen. Jedenfalls ist man bei Taubstummen ungleich mehr als bei Normalen auf die o bj e cti ve n Erscheinungen (Kopfneigung, Nystagmus) angewiesen.

$\mathrm{Zu}$ den Versuchen wurde eine kleine Chromsäure-Tauchbatterie von 18 Elementen angewendet, von denen gewöhnlich jedoch nur die Hälfte benutzt wurde. Der Strom wurde durch einen Schlüssel, Pohl'sche Wippe und Milli-Ampèremeter zu den beiden mit Leder überzogenen Knopfelectroden geleitet, diese mit Kochsalz gut durchfeuchtet auf die beiden Processus mastoidei der Versuchsperson aufgedrïckt. Die Stromstärke betrug, wenn der Kopf in den Strom eingeschaltet war, 5-10 M.-A., je nachdem Haut und Electroden mehr oder weniger gut durchfenchtet waren. Ich habe, ohne unangenebme Zufälle zu beobachten, die Stromstärke biş zu 
20 M.-A. steigern können, " und zwar geschah dieses in allen den Fällen, bei denen auf geringeren Strom keine Reáction erreicht werden konnte.

Zuerst experimentirte ich an den Schïlern des ProvinzialTaubstummen-Institnts. Bei dieser Untersuchung versäumte ich es, die untersuchten Kinder von den noch zu untersuchenden zu trennen, wodurch die Versuche bisweilen sehr gestört wurden, aber in ihren schliesslichen Resultaten nicht beeinflusst werden konnten, da ich den Strom bald von der einen, bald von der andern Seite in den Kopf eintreten liess, so dass willkürliches Taumeln sich immer verrathen musste. Von 65 Knaben and Mädchen. im Alter von 8-17 Jahren reagirten 5 nicht auf den galvanischen Strom, also $7,7 \%$. Unter diesen 65 waren 38 total taub, von letzteren versagten $4=10,5 \%$. Auf Nystagmus wurde leider bei diesen Versuchen nicht geachtet.

Bei der zweiten Schule, der Königsberger Vereins-Taubstummenanstalt, wurde die Anordnung des Versuches geändert. Vor allem wurden die untersuchten Schüler von den noch zu untersuchenden getrennt. Auch wurden diese Versuche bei geschlossenen Augen und einer Fussstellung vorgenommen, wie sie beim Turnen auf das Kommando „Füsse schliesst" eingenommen wird. Von den dortigen 76 Schülern und Schillerinnen im Alter von 6-16 Jahren versagten auf den galvanischen Strom $12=15,8 \%$. Unter den 76 waren 54 total tanb, von letzteren zeigten 10 keine Reaction = $18,5 \%$.

In demselben Jahre habe ich, um diese an Taubstummen gewonnenen Resultate mit den Befunden an ge s u n de n Kindern vergleichen zu können, die Schüler der hiesigen Steindammer Mittelschule mit dem galvanischen Strom untersucht. Die Versuchsanordnung war die gleiche wie bei der letzterwähnten Taubstummenanstalt. Untersucht wurden 78 Schüler im Alter von 9-14 Jahren. Von diesen reagirten 5 nicht auf den galvanischen Strom $=6,4 \%$. Diese Versager wurden am nächsten Tage noch einmal geprüft, indem ich mich selbst in den Strom mit einschaltete, und so das Vorhandensein und die Stärke des Stromes an meinen Empfindungen controlliren konnte. Das Resultat blieb das gleiche. Von diesen 5 Schïlern war keiner schwerhörig und hat keiner irgend eine Ohrenkrankheit durchgemacht. Die Versagenden unter den 
Taubstummen wurden auch noch einmal in der angegebenen Weise gepriff; ầ den Resultaten änderte sich dadurch nichts.

D́as Gesammtresultat der. Versuche aus dem Som me r 1892 war also:

von 141 Taubstummén versagen auf den galvanischen Strom $21=14,89 \%$,

von den darunter befindlichen 92 Totaltauben versagen $14=$ $15,21 \%$

von 78 normalen Kindern versagen $5=6,4 \%$.

Im Sommer 1893 habe ich die Untersuchung der Taubstummen wiederholt. Wenn auch bis dahin ein gewisser Wechsel unter den Schïlern stattgefunden hatte, d. b. die älteren die Anstalt verlassen hatten und jüngere dafür eingetreten waren, so war doch eine ziemlich bedeutende Anzahl mir vom vorigen Jahre bekannter Schtiler da. Meine Versuche erstreckten sich diesmal nicht allein auf den galvanischen Schwindel, sondern auch auf den durch Rotation hervorgerufenen Schwindel. Da letztere Versuche nicht direct an diese Stelle gehören, will ich nur kurze Mittheilung dariber machen. Auf dem Drehstuble ${ }^{1}$ ) befand sich ausser der Versuchsperson noch ein Beobachter, der durch Fingerauflegen auf die geschlossenen Lider der. Versuchsperson eventuelle Bulbusbewegungen bei der Rotation constatiren sollte. Derart batte $\mathrm{Kreid}$ seine Versuche angestellt und hatte gefunden, dass ca. $50 \%$ der Taubstummen diese reflectorischen Bulbusbewegungen vermissen liessen. Er bält diesen Nystagmus für ein Reagens der Functionen des Bogengangapparates. Bei meinen Versuchen wurden die Beobachtungen theils durch Herrn Dr. Mat thias, theils durch mich selbst ausgefiihrt. Es ergab sich, dass von. 167 Taubstummen $36=21,5 \%$ keinen Nystagmus zeigten. Von den 167 waren 122 total taub, von diesen zeigten $26=21,3 \%$ keinen Nystagmus. Von 53 untersuchten gesunden Schülern zeigten alle Nystagmus, 2 gar keinen Schwindel und 12 sehr schwachen Schwindel.

Bei den galva n is ch e n Untersuchungen wurde dieses Mal auch auf den Nystagmus geachtet, die Versuche also bei offenen

1) Derselbe ist nach den Angaben des Herrn Prof. Hermann sehr solide hergestellt und dreht durch Bewegung einer horizontalen mit. Kurbel versehenen Axe eine verticale Axe mit eiserner Platte, auf welcher ein Lebnstuhl mit den nöthigen Sicherungsvorrichtungen befestigt ist. 
Augen angestellt, sonst war die Anordnung so wie im Jahre vorher. Ich fand folgende Resultate bei den Sclüilern des Provinzial-Taıbstummen-Institutes: Von 88 Schülern zeigen keine galvanische Reaction $23=26,1 \%$, keinen Nystagmus $17=19,3 \%$; weder galvanische Reaction noch Nystagmus hạben $10=11,3 \%$. Ferner, diese Versuche mit den Rotationsversuchen vergliehen, ergibt sich, dass weder rotatorischen noch galvanischen Nystagmus von 88 Taubstummen 6 zeígen $=6,8 \%$, weder galvanische Reaction noch rotatorischen Nystagmus $8=9,1 \%$.

Von 78 Schülern der Vereins-Taubstummen-Anstalt versagten auf den galvanischen Strom $22=28,1 \%$, zeigten keinen galvanischen Nystagmus $13=16,7 \%$, beides zeigten nicht $8=10,3 \%$. Mit den Resultaten der Rotationsversuche verglichen, ergibt sich, dass weder galvanischen noch rotatorischen Nystagmus 4 zeigen $=5,1 \%$, weder galvanische Reaction noch rotatorischen Nystagmus $10=12,8 \%$.

Das Gesamm tresultat der Untersuehung a us d e m Jab re 1893 ist:

von 166 Taubstummen zeigen keine galvanische Reaction $45=27,1 \%$

keinen galvanischen Nystagmus $30=18,1 \%$,

weder galvanische Reaction noch Nystagmus $18=10,8 \%$,

weder galvanischen noch rotatorischen Nystagmus haben $10=6 \%$,

weder galvanische Reaction noch rotatorischen Nystagmus haben $18=10,8 \%$.

Vergleicht man diese Resultate mit denen des vorigen Jahres, so ergibt sich aus beiden, dass die Zahl der Versager eine verschiedene ist. Als Erklärung dafür den Wechsel unter den Schülern anzuführen, dürfte kaum genügen.

Ich ziebe aus dieser Ungleichbeit der Resultate den Schluss, dass allen diesen Versuchen ein gewisser Mangel an Objectivität anhaftet, dass der subjectiven Auffassung ein zu grosser Spielraum gelassen ist. Wer derartige Untersuchungen selbst gemacht hat, wird anerkennen muissen, auf wie grosse Schwierigkeiten man dabei stösst. Man bedenke, dass die Schüler der Taubstummeninstitute aus den ärmsten Familien stammen, dass in Folge ihrer Krankheit von den Eltern wenig Gewicht auf ihre Erziehung gelegt wird, dass sie von den Spielen ihrer Jugendgenossen grössten Theils ausgeschlossen 
sind, weil sie sich nicht mit ihnen verständigen können. Dass die geistige und körperliche Entwickelung aller dieser Kinder auf einer sehr niedrigen Stufe steht, ist klar,

Jedenfalls stimmen meine Resultate mit denjenigen Pollak's wenig überein. Er vermisst Kopfbewegungen bei $33 \%$, ich bei 14,89\% (erste Untersuchung) resp. 27,1\% (zweite Untersuehung). Augenbewegungen fehlten nach $\mathrm{Poll}$ a $\mathrm{k}$ bei $30,5 \%$, nach mir bei 18,1\%. Weder Augen- noch Kopfbewegungen zeigten bei Polla k $29,3 \%$, bei mir 10,8\%. Von Taubstummen, die bei $\mathrm{Kr}$ e id l auf der Drehscheibe keine Augenbewegungen zeigten, hatten bei Pollak nur etwa die Hälfte $(58 \%)$ keinen -galvanisehen Nystagmus, von 36 Taubstummen, die bei mir auf dem Drehstuhl keinen Nystagmus zeigten, hatten nur $10=27,8 \%$ keinen galvanischen $\mathrm{Ny}$ stagmus, es zeigten aber auch umgekehrt von 30 Taubstummen, die keinen galvanischen Nystagmus batten, nur $10=33,3 \%$ keinen rotatorischen Nystagmus.

Im Ganzen habe ich hiernach viel weniger galvanische Versager (um einen kurzen Ausdruck einzuführen) gefunden als Pollak; dieser fand 29,3\% totale Versager, ich nur $10,8 \%$. Normale Personen, wie ieh sie sehon vor der Pollak'schen Arbeit in grosser Zahl mit herangezogen habe, hat er gar nicht berïcksichtigt, und su kommt es, dass im gleichen Laboratorium $\mathrm{K} \mathrm{r}$ i d l ${ }^{1}$ j das Versagen eines normalen jungen Mannes als etwas Besonderes auffasst, und demselben einen Defect in inneren Ohre zuschreibt. Ich selbst babe unter 78 gesunden Schülern $6,4 \%$ galvanische Versager gefunden, was gar nicht so sehr weit binter der relativen Zabl der taubstummen Versager zurticksteht. Besonders bemerkenswertb aber ist das Verbältniss der galvanischen zu den rotatorischen Versagern. Wenn beide Reactionen, wie behauptet wird, von demselben Organ herrührten, so müssten, sollte man meinen, entweder beide Defecte stets vereinigt sein, oder wenigstens, falls eine der beiden Reactionen die schwieriger zu Stande kommende ist, diese nie ausbleiben, ohne dass aucb die andere fehlt. Statt dessen giebt es nach meinen Versuchen unter den Taubstummen sowohl galvanische Versager, welche rotatorische Reaction zeigen, als rotatorische Versager, bei welchen die galvanische Reaction vorhanden ist. Da ausserdem, wie schon mehr-

1) Centralbl. f. Physiol. Bd. 7. S. 165 . 
fach bemerkt (S. 213 f. und 226), derselbe Taubstumme, in Jahresfristen wiederbolt untersucht, mitunter eine friiher vermisste Reaction zeigt, so scheint mir der Schluss aus den Untersuchungen Taubstummer, dass die galvanische Reaction vom Labyrinth ausgeht, durchaus uicht auf festem Boden zu stehen. Am wenigsten aber kann ich es mitmachen, bei eimem Gesunden, welchem eine oder beide Reactionen fehlen, einen organischen Defeet zu diagnosticiren.

\section{Die Bedeutung des Vestibularapparates überhaupt.}

Der Gedanke, dass ein Theil desjenigen Organes, welches wir als Gebörapparat bezeichnen, einer ganz anderen Function dient, und dass wir ein Sinnesorgan besitzen, dessen wir uns, abweichend von allen anderen Sinnen, absolut nicht bewusst sind, hat etwas ungemein Widerstrebendes. Aber man muss zugeben, dass im Laufe der Zeit die stringenten Beweise, so weit es sich um Thiere handelt, in grosser Zahl erbracht sind. Die Versuche von Goltz, Breuer, Crum Brown, Ewald und vielen anderen sorgfältigen Experimentatoren miissen auch den äussersten Skeptiker zwingen, sich zu fügen. Freilich fehlt noch der erlösende Gedanke, nämlich der Nachweis des ursprünglichen inneren Znsammenhanges des statischen oder locomotorischen Sinnes mit der Gehörfunction. Denn ein solcher $\mathrm{m}$ u s $\mathrm{s}$ vorhanden sein; dafür spricht der innige Zusammenhang der Schnecke mit dem einen Otolithensack, sowie der gemeinsame Nerv beider Säcke, und da schneckenlose Thiere hören können, muss sogar der Vestibnlarapparat vielfach neben der neu entdeckten auch die Gehörfunction vollziehen können, oder allgemein mit vollziehen. Möglicherweise ist der primitive Zusammenhang in der phylogenetisch ältesten Lebensform, im Leben unter Wasser zu suchen. Die Wahrnehmung der eigenen Bewegung im Wasser, ohne jeden festen Stützpunkt, erfordert Sinnesorgane, welche durch das Medium oder durch die Bewegung der Körpermasse dislocirt werden. Ziemlich analog aber ist die Aufgabe, welche beim Hören vorliegt; die Vibrationen der Umgebung oder der Körpermasse können nur durch die Dislocation relativ fester oder relativ träger Körperbestandtheile zur Wahrnehmung gelangen.

Die Ueberzengung, dass vollständige Wegnahme der Laby- 
rinthe eine sebr starke Desorientirung der Thiere hervorbringt, welche besonders hervortritt," wenn man dem Thiere durch Verschluss der Augen und Anfstellung auf Watte seine übrigen Orientirungsmittel raubt, muss sich Jedem aufdrängen, der eine so operirte Taube beobachtet, und Herr Prof. Hermann, dem ich die vorstehende Betrachtung verdanke, jst durch die von Herrn Professor $E$ wald eingesandte labyrinthlose Taube, und später durch die hier operirten, von seinem früheren Standpunkte, welcher ungefähr mit dem noch vor Kurzem von $\mathrm{H}$ e n s e $\mathbf{n}^{1}$ ) vertretenen ubereinstimmte, zurïckgekommen, ohne sich im Uebrigen den von E w a 1 d entwickelten Theorien des ,Tonuslabyrintbes" anschliessen zu können.

Anders aber liegt die Sache heim Me nse he n. Aueb hier dem Lahyrinthe eine so fundamentale Bedentung zuschreiben $\mathrm{zu}^{*}$ wollen, wie bei der Taube, ist einfach unmöglich. Ich habe bei den vielen Untersuchungen Taubstummer auf galvanische und rota. torische Reaction, und bei dem damit verbundenen Verkehr mit vielen Taubst ummenlehrern meine Anfmerksankeit ganz besonders auf das generelle Verhalten der Taubstummen gericbtet. Dieselben zeiger, ausser der Taubstummbeit selbst und einer mit dieseñ Leiden, wie schon erwähnt, hăufig verbundenen geistigen Schwerfälligkeit, welche sich durch den Unterricht in der Taubstummenanstalt sichtlich vermindert, körperlich nichts, was auf Mangel eines anderen Sinnesorganes dentete. Dass sie in gewisser Hinsicht anffallend feinfühlig sind, ist schon angefiihrt (S. 213). Ihr Gang hat oft etwas Stampfendes oder Schliirfendes an sich; aber dies erkiatrt sich, abgesehen von dem Einfins, den geistige Schwerfälligkeit auch auf die körperliche Grazie hat, genúgend aus dem Umstande, dass șie ihre Tritte nicht bören, also gewisse nach dem Gebör zu erziehende Feinheiten des Auftretens nicht erlernen. Sie steben und bewegen sich sehr sicber auch mit verbundenen Augen; und was selur viel sagt, es sind unter ihnen auffallend viele ausgezeichnet gewandte Turner. Von erwachsenen Taubstumwen ist: ferner bekanat, dass sie grossentheils gern und gut tanzen. Wenn bei allen Taubstummen, welche auf dem Drehstuhl abnormes Verhalten zeigen, der Vestibularapparat feblt oder defect ist, so miusste man erwarten, dass wenigstens $d$ ie s e Taubstummen irgendwelche

1) Arch. f. Ohrenheilkunde Bd. 35. \$, 161. 
Haltungs- oder Bewegungsanomalien zeigen, und ich habe hierauf ganz besonders meine Beobachtung sowie meine Erkundigungen bei den Lehrern gerichtet. Alles fiel ziemlich negativ aus; indessen beruhigte ich mich hierbei nicht, mit Rücksicht auf die positiven Angaben von $\mathrm{Breuer}$ und von $\mathrm{Kr}$ eid $\mathrm{I}^{1}$ ). Nach letaterem können eine Arizahl Taubstumme mit geschlossenen Augen nicht auf einem Beine stehen, auf einem Rundholz laufen. Sebr wichtig ist hier die Vergleichung mit normalen Personen; Kreidl's. Bemerkung, dass ,alle die Aufgaben, die in dieser Weise an die Taubstummen gestellt wurden, von einem normalen Menschen mit Leichtigkeit geleistet werden konnten", besagt nichts darüber, ob entsprechende Prïfungen an einer grösseren Anzaht normaler Personen nit gescblossenen Augen angestellt wurden.

Herr cand. med. P. J u $\mathrm{n}$ ius hat auf Veranlassung des Herrn Prof. Herma n n zunächst eine Anzahl Schüler der beiden hiesigen Taubstummenanstalten auf ihr Verhalten untersucht, nämlich 16 Knaben und 10 Mädchen aus der Provinzial- und 16 Knaben aus der Vereins - Anstalt, im Ganzen 42. Die Prüfung erstreckte sich auf gewöhnliches Stehen, Stehen auf einem Bein, Geben, und zwar alles mit offenen und mit verbundenen Augen. Selbst die geringste Abweichung vom normalen Verhalten wurde als Abnormität verzeichnet; die Ergebnisse sind also so u n g i $\mathrm{n}$ s ti $\mathrm{g}$ wi e $\mathrm{m} \ddot{\mathrm{g}} \mathrm{g}$ i c b gedeutet. Folgende kleine Tabelle stellt dieselben dar $^{2}$ ):

\begin{tabular}{l|c|c|c}
\hline & Stehen & $\begin{array}{c}\text { Stehen auf } \\
1 \text { Bein }\end{array}$ & $\begin{array}{c}\text { Gradeaus- } \\
\text { Gehen }\end{array}$ \\
\hline & 0. & 0. & 0. \\
Normal & $41=97,6 \%$ & $32=76,2 \%$ & $38=90,5 \%$ \\
Abnorm & $1=2,4 \%$ & $10=23,8 \%$ & $4=9,5 \%$ \\
& G. & G. & G. \\
Normal & $39=92,9 \%$ & $16=38,1 \%$ & $7=16,7 \%$ \\
Abnorm & $3=7,1 \%$ & $26=61,9 \%$ & $35=83,3 \%$
\end{tabular}

Unter den untersuchten Schiilern sind mebrere erst soeben in die Anstalt eingetreten, und diese zeigen entschieden in jeder Hinsicht das ungünstigste Verbalten.

1) Dies Archit Bd. 51. S. $144 \mathrm{ff}$.

2) 0 . bedeutet offene, G. geschlossene Augen. 
Zum Vergleiche wurden 16 gesunde Knaben aus einer Volksschule auf ihr Verhalten geprüft, wobei sich ergab:

\begin{tabular}{|c|c|c|c|}
\hline & Stehen & $\begin{array}{c}\text { Stehen auf } \\
1 \text { Bein }\end{array}$ & $\begin{array}{c}\text { Gradeaus: } \\
\text { Gehen }\end{array}$ \\
\hline $\begin{array}{l}\text { Normal } \\
\text { A bnorm }\end{array}$ & $\begin{array}{c}0 \\
16=100 \%\end{array}$ & $16=\begin{array}{l}0 \\
-100 \%\end{array}$ & $16=100 \%$ \\
\hline $\begin{array}{l}\text { Normal } \\
\text { Abnorm }\end{array}$ & $\begin{array}{c}\mathrm{G} . \\
16=100 \%\end{array}$ & $\begin{array}{c}\mathrm{G} . \\
10=62,5 \% \\
b=37,5 \%\end{array}$ & $\begin{array}{c}\mathrm{G} . \\
7=43,7 \% \\
9=56,3 \%\end{array}$ \\
\hline
\end{tabular}

Auf den ersten Blick scheinen diese Uebersichten die Ansicht Kreidl's zu bestätigen; die Procentzablen der Abnormen sind durchgehends bei den Taubstummen höher als bei den Normalen. Wenn man aber sieht, dass auch von ganz gesunden Knaben ï be r d i e H älf te mit verbundenen Augen nicht gradeaus gehen, und $\ddot{u} b e r$ e in Drittel nicht ordentlich auf einem Beine stehen können, so wird man schwerlich greneigt sein, die Abweichungen anf den Mangel desjenigen Sinnesorgans zu beziehen, welches den Taubstummen grossentheils fehlen soll. Viel wahrscheinlicher ist es doch, dass die schon erwähnten erzieblichen Wängel, und die grössere Aengstlichkeit und Befangenheit der Taubstummen die Ursache der Verschiedenheit sind.

Wie unwahrscheinlich ist es feruer, dass ein Organ des Kopfes für die Künste des Balancements auf den Füssen von entscheidender Bedeutung sein soll. Hier kommt es darauf an, geringe Abweichnngen des Scbwerpunkts von dem Verticalıanm über der Unterstiitzungsfläche sofort wahrzunehmen und zu corrigiren. Solehe Abweichungen bewirken gar keine merkliche Drehungs. componente des Kopfes, zu deren Wahrnehmung das fragliche Organ geeignet wäre. Vielmebr ist längst bekannt, dass die zur Balancirung erforderlichen Wahrnehmungen wesentlich dem Tastsinn der Fusssolilen und vielleicht den Empfindungen der Gelenke zufallen, dass deswegen Tabische mit geschlossenen Augen nicht stehen können. Der Vestibularapparat nützt also ganz offenbar diesen Kranken für die in Rede stehende Leistung gar nichts! Kreidl's Ansicht, dass das Balancement durch den Vestibularapparat vermittelt werde, kann daher nicht richtig sein.

Worin besteht also der Nutzen des Organs für den Menschen? 
Wir wollen zugeben, dass es vermuthlich den Drehschwindel hervorbringt; in der That seheint dies die einzige positive Empfindung zu sein, durch welehe sich der Besitz dieses Sinnesorganes uns verräth. Aber die Fähigkeit sehwindlig zn werden, kann nicht gut als ein Vortheil der Organisation angesehen werden, es sei denn, dass der Schwindel uns zur Warnung dient, Drehbewegungen, welche vielleicht Gefahren bergen, nicht ïber einen gewissen Grad oder eine gewisse Dauer anwachsen zu lassen ${ }^{1}$ ). K reidl schreibt dem Vestibularapparat noch eine andere Leistung $z u$, deren Nutzen für die Organisation noch viel schwerer verständlich ist. Der Defect des Organs soll nämlich das Bewusstsein der resultirenden Richtung aus Schwer- und Centrifngalkraft verhindern²). Nach ihm wäre aber: der Defectuöse gegenüber dem Normalen geradezu im Vortheil, denn ersterer erkennt auf dem Carroussel die absolute Verticalrichtung richtig, der Normale wird durch die Centrifugalkraft getäuscht!

Aber grade dieses Erkennen der Verticalrichtung seitens des Taubstummen ist so unbegreiflich, dass man den Versuchen gegenüber nisstrauiseb wird. Er kann doch unmöglich eine Erinnerung oder eine verwendbare Gewohnbeit mitbringen, sondern ist während der Drehung auf seine Sinnesorgane allein angewiesen. Er befindet sich aber in einer Situation, in welcher nun einmal die Richtung der. Massenbeschlennigung von der Verticalen gesetzmässig abweicht und in je d e r Hinsicht, für die Sinne so gut wie fuir ein Glas Wasser, in die Resultante aus Schwer- und Centrifugalkraft fällt. Entweder hat der Taubstumme noch Sinne übrig, um diese Richtung zu erkennen, dann muss er schief einstellen, oder er bat keine übrig, dann müsste er gar nicht oder sinnlos einstellen. Wie er dazu kommt auf die absolute Verticale einzustellen, ist unbegreiflich, und der Versuch kann daher nicht in $\mathrm{K} r$ e i d l's Sinn acceptirt werden.

Alles zusammenfassend kommt man zu der Ueberzeugung, dass der sog. statische Sinn des Labyrinthes beim Menschen nur eine äusserst geringe Bedeutung hat. Das geht auch schon daraus

1) James (a. a. O.) will merkwürdigerweise auch Fehlen des sog. Höhenschwindels, einer offenbar rein intellectuellen Erscheinung, bei relativ vielen Taubstummen beobachtet haben.

2) Dies Archiv Bd. 51 . S. $133 \mathrm{ff}$. 
hervor, dass er nicht schon vor Jahrhunderten durch Taubstummenbeobachtnng entdeckt ist, sondern dass er erst nach den experimentellen Entdeckungen an der Taube mïhsam und spurweise auch am Menschen durch zweifelhafte Mängel Taubstummer bestätigt werden konnte.

Es könnte sich mit diesem Sinne ähnlich verhalten wie mit dem Geruchssinn, der ebenfalls beim Mensehen, wenn auch ungleich mehr als der statische Sinn, doch im Vergleich zu den Thieren nur sehr wenig entwickelt ist. Hier aber giebt sich die geringere Entwicklung auch morphologisch am Lobus olfactoriusdes Gehirns, am knöchernen Nasenlabyrinth und an der Ausdehnung und Ausstattung der Riechhaut zu erkennen, während das Ohrlabyrinth in seiner Entwicklung beim Menschen durehaus nicht zuriucksteht. Man darf wohl auch hieraus schliessen, dass der Vestibularapparat durchweg auch Hörfunctionen hat ( $\mathrm{vgl}$. oben S. 228). Ja man könnte sogar vermuthen, dass er $\mathrm{z} \mathrm{u} \mathrm{m} \mathrm{Höre} \mathrm{n}$ unentbehrlich ist, d. h. dass der Besitz der Schnecke zum Hören nicht genügt; denn nach $M y g$ ind ${ }^{1}$ ) zeigt e i n F iu $\mathrm{f}$ telaller Taubsummen nur anden Bogengängen Abnormitäten.

Anch bei Fischen and Amphibien spielt offenbar die bei der Taube so stark entwickelte statische Labyrinthfunction nur eine relativ geringe Rolle neben der Hörfunction. Wie viele Autoren konnten bei solehen Thieren nach Exstirpation der Bogengänge oder des ganzen Labyrinthes keine Haltungs- und Bewegungsstörungen constatiren! Da wir vom Verbalten der Reptilien kaum etwas wissen, so darf man sagen, dass überhaupt $\mathrm{n} \mathrm{u} \mathbf{r}$ bei V ö g e ln eine erhebliche Bedeutung des uns beschäftigenden Sinnes nachgewiesen ist; ihnen am nächsten scheinen die Fische zu stehen. Beide Thierklassen bewegen sich in ihrem Medium abweichend von den nur kriechenden und gehenden Thieren, nach d r ei Dimensionen ohne feste Stütze, und man kann sich wohl eine Vorstellung davon machen, dass bei ihnen ein besonderes Orientirungsorgan wichtiger ist als bei anderen Wirbelthieren, und sich daher mehr ausgebildet oder weniger zuriuckgebildet hat als bei den übrigen.

1) H. Mygind, Taubstummheit. Berlin u. Leipzig 1894. S, 158. 


\section{Nachtrag.}

Die Abfassung der vorstehenden Arbeit ist in ihren wesentlichen Theilen schon vor dem Erscheinen einiger neueren, denselben Gegenstand betreffenden Publicationen erfolgt, auf welche daher nur anhangsweise kurz eingegangen werden kann.

A. B r u $\mathrm{k}^{1}$ ) hat nicht allein Taubstumme, sondern wie ich, resp. Herr $J$ u $\mathbf{n}$ i us, auch Normale auf gewisse Bewegungsanomalien untersucht, und unter ersteren etwa $50 \%$, unter letzteren nur $81 / 3 \%$ mit solchen gefunden. Wenn auch unsre Ergebnisse an Normalen mit geschlossenen Augen ungünstiger waren, so ist doch die Differenz nicht so beträchtlich, dass B r u c k's Versuche nnser Urtheil in Frage stellen könnten. $\mathrm{Br}$ u ck gibt zwar an, dass die mangelhaft fnnetionirenden nicht tanbstummen Kinder schwäcblich und ängstlich waren (bei unseren Versuchen wurde eine solche Beziehung durchaus nicht bemerkt), sagt aber selbst, dass diese Mängel die Abweichungen nicbt erklären, und dass "es auch vielen von uns nicht immer möglich ist, bei geschlossenen Augen in grader Richtung vorwärts zu gehen." Auch will B ru ck die Störungen nicht rom liangel eines specifischen Balancirapparates, sondern von allgemeinen durch den Hördefect und sonstige mit der Taubstummheit zusammenhängende Uebelstände bedingten Vängeln herleiten, eine Ansicht, welche sich mit der hier vorgetragenen im Wesentlichen deckt.

Die interessante Arbeit von L. W. St e r $\mathrm{n}^{2}$ ) ist, abgesehen von ihrer Bedeutung für die Pathologie der Taubstummeit, für unsre Frage in sofern von Bedeutung, als ans derselben ein Zusammenbang zwischen Locomotions- und Sprechfähigkeit der Taubstummen sich zu ergeben scheint. S $t$ e $r$ ist geneigt, dies mit der Ewald'schen Idee des "Tonuslabyrinthes" in Zusammenhang zu bringen. Näher liegt es wohl, im Anschluss an das S.231 Gesagte anzunehmen, dass die mit der Taubstummheit häufig verbundene allgemeine Ungeschicklichkeit allen Defecten, welche ausser der Taubheit beobachtet werden, zu Grunde liegt, und schwierigere Leistungen, wie Balancirkiunste und Sprechen, minderwerthig macht.

Zum Schluss sei es mir gestattet, meinem hochverebrten Lehrer, Herrn Geheimrath H e r m a n n für seine mir bei der Arbeit stets zu Theil gewordene Unterstuitzung meinen herzlichsten Dank anszusprechen.

1) Dies Archiv Bd.' 59, S. 16.

2) Dies Archiv Bd. 60. S. 124. 